\title{
Novel immunological tolerance of human cancer cell xenotransplants in zebrafish
}

Beibei Zhang ${ }^{1 \dagger}$, Yasuhito Shimada ${ }^{1-5 \dagger}$, Tomokazu Hirota ${ }^{1}$, Michiko Ariyoshi ${ }^{1}$, Junya Kuroyanagi ${ }^{1}$, Yuhei Nishimura ${ }^{1-5}$, and Toshio Tanaka ${ }^{1-5 \S}$

1 Department of Molecular and Cellular Pharmacology, Pharmacogenomics and Pharmacoinformatics, Mie University Graduate School of Medicine, Mie, Japan

${ }^{2}$ Department of Systems Pharmacology, Mie University Graduate School of Medicine, Mie, Japan

${ }^{3}$ Mie University Medical Zebrafish Research Center, Mie, Japan

${ }^{4}$ Department of Bioinformatics, Mie University Life Science Research Center, Mie, Japan

${ }^{5}$ Department of Omics Medicine, Mie University Industrial Technology Innovation Institute, Mie, Japan

${ }^{\dagger}$ Contributed equally to this work

${ }^{\S}$ Corresponding author

Toshio Tanaka, MD, PhD, Department of Molecular and Cellular Pharmacology, Pharmacogenomics and Pharmacoinformatics, Mie University Graduate School of Medicine, 2-174 Edobashi, Tsu, Mie 514-8507, Japan

Tel: $+81-59-231-5411$

Fax: $+81-59-232-1765$

E-mail: tanaka@doc.medic.mie-u.ac.jp 


\title{
Running head
}

Tolerance in zebrafish xenotransplantation

\begin{abstract}
Abbreviations

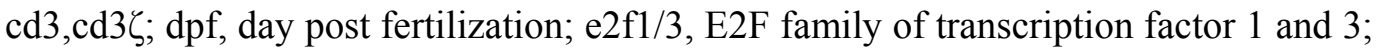

foxp3, forkhead box P3; hpf, hour post fertilization; il-17, interleukin 17; ifn- $\gamma$, interferon gamma; IFNG, interferon gamma; klf1, Kruppel-like factor 1 (erythroid); KOr, Kusabira-Orange; mpf, month post fertilization; myod1, myogenic differentiation 1; MEK1/2, mitogen-activated protein kinase kinase 1/2; SNEA, subnetworkenrichment analysis; STAT5A, signal transducer and activator of transcription 5A; tbx20, T-box 20
\end{abstract}




\begin{abstract}
Immune deficiency orsuppression in host animals is an essential precondition for the success of cancer cell xenotransplantation, because the host immune system has a tendency to reject implanted cells. However, in such animals, the typical tumour microenvironment seen in cancer subjects does not form because of the lack of normal immunity. Here, we developed a novel zebrafish(Daniorerio) model based on two-rounds of cancer cell xenotransplantation that achieved cancer-specific immunological tolerance without immunosuppression. We irradiated human cancer cells(PC-3, K562 andHepG2)to abolish their proliferative abilities and implanted them into zebrafishlarvae. These cells survived for 2 weeksin the developing host. Three months after the first implantation,the zebrafish were implanted with the same, but non-irradiated,cell lines. These cancer cells proliferated and exhibited metastasis without immune suppression. To reveal the transcriptional mechanism of this immune tolerance, we conducted dual RNA-seq of the tumour with its surrounding tissues and identified several regulatory zebrafishgenes that are involved in immunity; the expression of plasminogen activator,urokinase and forkhead box P3 were altered in response to immunological tolerance. In conclusion, this xenograft method has potential as a platform for zebrafish-based anti-cancer drug discovery because it can closely mimic human clinical cancers without inducing immune suppression.
\end{abstract}




\section{Introduction}

Xenotransplantation of human cancer cells provides a valuable model for pathophysiological and therapeutic studies in cancer research. One challenge is the avoidance of immune rejection by the host animal. Induction of immune deficiency, for example using the NOD/SCID mouse[1], $\gamma$-irradiation, or immune-suppressing agents[2], has been used to facilitate xenotransplantation. Although these models have contributed greatly to the understanding of human cancers, the lack of systemic immunity, mainly $\mathrm{T}$ and $\mathrm{B}$ cell function, is a problem for further understanding of cancer mechanisms[3, 4]. Correspondingly, the drug response in cancerxenografted "nude" animals does not always correlate to the clinical situation owing to the changes in the immune system [5]. In addition, the immune suppression treatments can induce spontaneous tumours in the host, thus confounding xenotransplantation cancer studies [6].

Zebrafish(Daniorerio) have been successfully used for decades as a model organism for preclinical studies and drug discovery. In cancer research, cancer xenograftzebrafish represent a promising alternative model to study tumour proliferation[7, 8], angiogenesis $[9,10]$, metastasis $[11,12]$ and cancer stemness[13, 14]. To avoid immune rejection of the xenograft, cancer cell implantationhas been

performedat the embryonic stage (24-72 hours post-fertilization, hpf) when the adaptive immune response is not established.Although the sites of haematopoiesis are different between zebrafish and mammals, the cellular and regulatory processes of haematopoiesis,includingfor immune cells, are highly conserved[15, 16]. In fact, most of the immune cells found in mammals have also been identified in zebrafish[17, 18$]$. Because of this similarity, the number of studies examining adult-zebrafishxenografts is small. Gamma-irradiation [19] and immune-suppressants [20]have been used to 
ablate immune cells of zebrafish to establish adult-zebrafishxenografts. Recently, Tang et al. developed an immuno-compromised zebrafish model using rag2 mutants, which allows long-term engraftment of human cancer cells [21]. In spite of these successful studies, such methodologies are limited owing to the lack of a normal immune system in the host.

In this study, we established a novel cancer xenograftzebrafish with immunological tolerance specific to the implanted cancer cells. The implanted cancer cells proliferated and exhibited metastasis without host immune suppression. 


\section{Materials and Methods}

\section{Ethical approval}

The animal experiments in this manuscript conformed to the ethical guidelines established by the Institutional Animal Care and Use Committee of Mie University, which follow the NIH guidelines (Guide for the Care and Use of Laboratory Animals).

\section{Cell culture and fluorescent labelling}

PC-3 cells (human prostate cancer), K562 cells (human chronic myelogenous leukaemia) and HepG2 cells (human hepatocarcinoma) were obtained from the RIKEN Cell Bank (Tokyo, Japan). The methods for cell culture and fluorescent protein labelling were performed as in a previous study [22]. We denoted KusabiraOrange protein-expressing cells as PC3-KOr, K562-KOr and HepG2-KOr, respectively.

$\gamma$-Irradiation of cancer cells

PC3-KOr, K562-KOrand HepG2-KOr cells were cultured in $25 \mathrm{~cm}^{2}$ flasks containing $10 \mathrm{ml}$ medium at a density of $4 \times 10^{5}$ cells $/ \mathrm{ml}$. Irradiation was delivered from a cobolt-60 gamma irradiation using a CAX-150-20 (ChubuMedical, Aichi, Japan). We prepared 10 Gy-irradiated PC3-KOr, 6 Gy-irradiated K562-KOr and 10 Gy-irradiated HepG2-KOr cells, all of which lacked proliferative capacity but did not die.We denoted the above irradiated cells as Ir-PC3-KOr, Ir-K562-KOr, and IrHepG2-KOr, respectively. 


\section{Zebrafish}

Care and breeding of zebrafish were performed following protocols as described by Westerfield[23]. nacre/rose/fli1:egfpzebrafish were used because they lack pigmentation and are largely transparent, which facilitates in vivo monitoring of tumours[24].

Cancer cell xenotransplantation intozebrafish embryos

The xenotransplantation procedures used are described in our previous studies[24]. In brief, just before xenotransplantation, 48 hpfzebrafish were anaesthetized with 2-phenoxyethanol solution (100 ppm; Wako Pure Chemical Industries, Osaka, Japan), and arrayed on a holding sheet. Cancer cells $\left(1 \times 10^{6}\right.$ cells $)$ were suspended in $30 \mu$ LMatrigel (BD Biosciences, San Jose, CA, USA). The avascular region of the yolk sac was then injected with $10 \mathrm{nl}$ of the cell suspension containing 100-200 cells using a glass needle and FemtoJet injection system (Eppendorf, Hamburg, Germany). For control fish, $10 \mathrm{nl}$ of Matrigel was injected into the yolk sac.

\section{Cancer cell xenotransplantation into adult zebrafish}

Cancer cells were suspended at 3,000 cells/ $\mu \mathrm{L}$ in Hanks' balanced salt solution. One microliter of cell mixture was injected into a site near the dorsal aorta of each recipient fish using a $10 \mu \mathrm{L} 701 \mathrm{~N}$ syringe (Hamilton, Reno, NV, USA). Injected fish were placed in a recovery tank for $1 \mathrm{~h}$ and then returned to the tank system at $34^{\circ} \mathrm{C}$. 
Imaging

Zebrafish were anaesthetised with 2-phenoxyethanol solution (100 ppm; Wako Pure Chemical Industries). Images were captured using a MZ16F stereoscopic microscope (Leica Microsystems, Wetzlar, Germany) equipped with a DP71 digital camera (Olympus, Tokyo, Japan).For fluorescence imaging, GFP2 (for fli1:egfp) and DsRed (for KOr) filters were selected. Cancer cell proliferation was calculated by the ratio of KOr fluorescence intensity in the tumour area relative to that at day 0using ImageJ software (National Institutes of Health, Bethesda, MD, USA) as described previously $[10,22,25]$.

RNA extraction, $c D N A$ synthesis, and $q-P C R$

Total RNA was extracted from cells or tissues using the RNeasy Mini kit (Qiagen, Hilden, Germany) according to the manufacturer's instructions. The firststrand cDNA was synthesised from 200 ng of total RNA using the SuperScript III cDNA synthesis kit (Life Technologies) with random primers (Life Technologies). Quantitative polymerase chain reaction (q-PCR) was performed using Power SYBR Green Master Mix (Applied Biosystems, Foster City, CA, USA) and a 7300 real-time PCR system (Applied Biosystems) as recommended by the manufacturer. The primers shown in Table S1 were used to amplify target genes.

RNA-seq

Total RNA was extracted from tumours and their surrounding tissuesof adult stage PC3-KOr xenografts (7dpi) with or without Ir-PC3-KOr embryonic implantationusing the RNeasy Mini kit (Qiagen) according to the manufacturer's instructions. Adult PC3-KOr xenografts without Ir-PC3-KOr embryonic implantation 
were a control group. Each group (Ir-PC3-KOr and control) contained three fish. Before library construction, ribosomal RNA was depleted using a Ribo-Zero Magnetic Kit (Epicentre, Madison, MI, USA) according to the manufacturer's instructions. RNA libraries were then constructed using an Ion Total RNA-Seq Kit v2 (Life Technologies) according to manufacturer's instructions. The libraries were then sequenced to generate dual RNA-seq data using Ion Proton (Life Technologies).

\section{Bioinformatic analysis of dual RNA-seq}

Dual RNA-seq data were mapped and tag counts were performed using Genomics Workbench (CLC bio, Aarhus, Denmark). Then, we performed gene expression analysis using the R package and Tag Count Data Comparison (TCC). The package incorporates multi-step normalisation methods, whose strategy is to remove potential genuine differentially expressed genes before performing data normalisation [26]. After statistical tests, we performed further bioinformatic analyses (gene-set enrichment analysis (GSEA) and subnetwork enrichment analysis (SNEA) using Pathway Studio 9.0 (Elsevier, Amsterdam, Holland) according to the developer's instructions.

\section{Statistical analysis}

Data were analysed using statistical package SPSS 19.0 (SPSS, Chicago, IL, USA). Differences between two groups were examined for statistical significance by independent Student's t-test. For multiple comparisons, assessments were analysed using one-way analysis of variance (ANOVA) followed by the Bonferroni-Dunn multiple comparison test. All data are presented as the mean \pm SEM. Values of $P<$ 0.05 were considered statistically significant. 


\section{Results}

Implantation of irradiated human cancer cells induces immunological tolerance.

The key distinction of our human cancer cell xenotransplantation model is the double xenotransplantation strategyat embryonic and adult stages (Fig. 1A). We implanted Ir-PC3-KOr or naive (not irradiated) PC3-KOr cells into 48 hpflarvae (Fig.1B).Seven days after implantation, the Ir-PC3-KOr cells had almost disappeared from the xenograft, while PC3-KOr cells were still presentand remained until death of the hosts in the subsequent days (Fig.1B).After 1 month, almost all PC3-KOr xenograftedzebrafish were dead, while about $70 \%$ of Ir-PC3-KOr xenograftedzebrafish survived and grew normally without any appearance of the implanted cells. When the embryonic Ir-PC3-KOr xenograftedzebrafish and their littermates (control group without cancer cell implantation) reached 3 months post fertilization (mpf), we conducted xenotransplantation of naive PC3-KOr cells. Seven days after implantation at this adult stage,the Ir-PC3-KOr group exhibited a larger tumour size than the control group (Fig. 1C, left panels). Additionally, the Ir-PC3-KOr group developed metastasis in the tail region; however, none of the control zebrafishtails exhibited obvious fluorescence from cancercells (Fig. 1C, right panels and Fig. 1D). In the following days, the tumours continued to proliferate whilethe tumours almost disappeared from control zebrafish(Fig. 1E).

To validate this novel method for xenotransplantation, we conducted the same experiments with human chronic myelogenous leukaemiaK562 cells (Supplementary Fig.S1)and hepatocarcinoma HepG2 cells (Supplementary Fig. S2). K562-KOr and HepG2-KOr xenografts exhibited the same results as PC3-KOr xenotransplantation. These results demonstrate that this protocol should be applicable to the majority of human cancer cell lines. 


\section{Ir-K562-KOr xenograftedzebrafish reject PC-3 cell xenotransplantation}

To evaluate the selectivity of the immunological tolerance induced by cancer cell implantation into embryos, we performed cross-xenotransplantationwith PC3-KOr cellsinto $3 \mathrm{mpf}$ adult zebrafish that received embryonicIr-K562-KOr xenografts. After 7 days, PC3-KOr cell implantsproduced smaller tumours than K562-KOr cells $(P<$ 0.05; Fig. 2A and Supplementary Fig. S3) and these tumours were almost disappeared by day 14. KOrmRNA was only expressed in implanted cancer cells; therefore, its expression in the blood could be an indicator of circulating cancer cells[24]. We foundthat $\mathrm{KOr} m R N A$ levels in the blood of PC3-KOr xenograft fish(cross-xenograft with embryonic Ir-K562-KOr) were significantlydecreased in comparison with K562KOr xenograft fish on day $28(P<0.05$; Fig.2B).

\section{Transcriptome analysis of the immunologically tolerant zebrafish}

The forkhead box P3 (FOXP3) gene, a biomarker expressed in regulatory $\mathrm{T}$ cells $\left(\mathrm{T}_{\text {reg }}\right)$, plays a key role in immune modulation[27], a process central to immunological tolerance. It has been established that the cell population expressing FOXP3 plays a significant role in inducing and maintaining immunological tolerance in human and model animals $[28,29]$. Zebrafishfoxp 3 has also been characterized and was found to have a similar role to that in mammals, although $T_{\text {reg }}$ cells in zebrafish have yet to be purified [30]. Therefore, in this study, we examined the foxp 3 gene as a marker of functioning $\mathrm{T}_{\text {reg }}$ cells. Zebrafishfoxp 3 mRNA levels in the blood ofIr-PC3KOr xenograft fishwere higher than that of control fish beforeand also after the second xenotransplantation $(P<0.01$; Fig.3A).We also foundthat zebrafishfoxp3 expression was higher in the tumour area than in the non-tumour tissue $(P<0.01$; 
SupplementaryFig.S4). In contrast to foxp3 expression in the blood, after the second xenotransplantation, the expression of genes related to inflammation $(c d 3 \zeta[c d 3]$, interferon gamma[ifn- $\gamma]$ and interleukin 17[il-17]) was increased to a lesser degree in immunological tolerant fish compared with the control fish that did not receive the first xenotransplantation at their embryonic stage $(P<0.01$; Fig. $3 \mathrm{~B})$.

To further reveal the mechanism of cancer progression in this model, we conducted RNA-seq analysis of the Ir-PC3-KOr xenografts. We collected tumours and their surrounding tissues at 7 days after the adult stage xenotransplantation and performed next generation sequencing. Adult PC3-KOr xenograft fish without embryonic Ir-PC3-KOr implantation,which still had tumours, were used as a control.Using GSEA [31], we found that 32 human genes and 627 zebrafish genes were significantly $(P<0.05)$ altered in Ir-PC3-KOr xenograftedzebrafish compared with control fish that did not receive the first xenotransplantation at the embryonic stage. SupplementaryTable S2shows the list of genes with the greatest GSEA results. GSEA results of the human transcriptome showed alteration of some immune-related gene sets including those involved in the inflammatory response; however, these effects were not observed in thezebrafishtranscriptome. To increase the sensitivity of the analysis, we performedSNEA[32] of the human and zebrafish RNA-seq data to identify key molecules regulating the expression of the genes that showed significant alteration (false discovery rate $<0.05$ ). Table 1 shows pathways involved in this cancer xenograft in both human cancer cells and the zebrafishhost.In humans, mitogen-activated protein kinase kinase 1/2 (MEK1/2), interferon gamma (IFNG) and signal transducer and activator of transcription 5A (STAT5A) were predicted to be involved in tumour proliferation. In zebrafish, Kruppel-like factor 1 (erythroid) (klfl), myogenic differentiation 1 (nyod1), T-box $20(t b x 20)$ and E2F family of transcription 
factor 1 and 3 (e2fl/3) were predicted to be involved in host immunity. Expression of genes involved in these zebrafishpathwayswas confirmed by q-PCR (Fig.3C).

\section{Discussion}

Since the discovery in 1945 of immune-genetic interactions during vascular anastomoses in experiments involving a set of bovine twins [33], studies concerning immunological tolerance induced at the embryonic stage by artificial intervention have been performed in rat [34], chicken [35] and Syrian hamster [36]. These animal models had intact immune systems (were not nude animals) and the xenografted cancer grew and killed the host within arelatively short period, which made it difficult to study cancer progression or drug responsesin adulthood.

The zebrafishimmune system is similar to that of mammals in that they both have innate and adaptive immune systems [37]. Meanwhile, astudy of lipopolysaccharidestolerance suggested the possibility that immune tolerancecan be inducible in zebrafish[38]. In addition, human malignant melanoma cells implanted into zebrafish embryos were shown to persist until the adult stage (3 mpf), but could not form a tumour mass [39]. These studies indicated that xenotransplantation at the embryonic stage could impart immunological tolerance to implanted human cells.

A key distinction of our xenotransplantation model is the double xenotransplantation strategy, which avoids cancer-induced death following the first implantation in the early developmental stages. At 48 hpf, a viable stage for the first cancer implantation, zebrafish embryos begin to develop an innate immune system; however, this stage precedes thymus development [40], resulting in the avoidance of immune rejection of implanted cells, as observed in this study. Xenotransplanted cancer cells usually proliferate in immune-compromised animals and finally kill the host; however, our irradiated cells disappeared and had no effect on the host survival 
ratio. Furthermore, theyinduced specific immunological tolerancefor the implanted cells. This methodology was applicable to not only human prostate cancer (PC-3) but also to CML (K562) and hepatocarcinoma (HepG2) cells, suggesting that this phenomenon is common to most kinds of human cancer cells. Additionally, the naive cancer cells without irradiationimplanted intoembryonic control fishpersisted for more than 12 days and finally killed the host, similar to results in rodent studies [34]. These results indicate that the zebrafish immune system can recognize the antigenicity of each cancer cell line. Theseresults were similar to those ofanother study that examined LPS response in zebrafish[38]. Moreover, zebrafish with immunological tolerance for a particular cell type still rejected other cell types that are cross-xenotransplanted, which suggests that they maintain immune tolerance, but not immune deficiency.

The induction of immune tolerance in xenografts may require several steps, especially deletion, anergy and the emergence of $T_{\text {reg }}$ cells. $T_{\text {reg }}$ cells induce and maintain immunological tolerance by inhibiting effector $\mathrm{B}$ and $\mathrm{T}$ cells[28], which promote autoimmune diseases [41] and tumour progression[42]. Thus, $\mathrm{T}_{\text {regcells are an }}$ important therapeutic target, and inhibition of their activity or numbers is thought to enhance host-derived immunological attack against cancer cells[43, 44]. $\mathrm{T}_{\text {reg }}$ cells express FOXP3[27], a gene that is also present and functional in zebrafish[30]. However, zebrafish $\mathrm{T}_{\text {reg }}$ cells have not yet been identified and purified. Our results demonstrated the involvement of zebrafishfoxp3 in immunological tolerance during cancer progression, similar to findings in mammals.

Dual RNA-seq analysis was first used in zebrafishto analyse the pathogen-host interaction of infectious diseases [45]. While protein sequences are similar between human and zebrafish, RNA sequences tend to be different. Thus, deep sequencing is appropriate for transcriptome comparisons in mixed-species tissues, while DNA 
microarrays are of limited utility. In the human transcriptome, pathways involving mitogen-activated protein kinase 1/2 (MEK1/2), interferon gamma (IFNG) and signal transducer and activator of transcription 5A (STAT5A) were activated during cancer progression. For the MEK 1/2 pathway, aberrant signalling through the RAS/RAF/MEK pathway leads to cell transformation [46], and several MEK inhibitors are now under clinical trials against many types of cancer [47]. This suggests that malignant transformation would continue in the implanted human cancer cells. Interferon- $\gamma$ (IFNG) is a cytokine whose biological activity is conventionally associated with cytostatic / cytotoxic and anti-tumour mechanisms during the cellmediated adaptive immune response [48]. IFNG regulates solute carrier family 7 , member 7 (SLC7A7), whichwas up-regulated in our study and induces immunosuppression in tumour-infiltrating lymphocytes [49]. SLC7A7 is a marker for glioblastoma prognosis and is involved in malignant transformation [50]. STAT5A is highly expressed in human prostate cancer cells and promotes metastasis [51], and is thus considered a promising therapeutic target against prostate cancer $[52,53]$.In the zebrafishtranscriptome, we found that urokinase-plasminogen activator (plau) downregulation occurred in the $e 2 f 1$ and $e 2 f 3$ sub-networks, which has been reported to induce TGF- $\beta$ activation, thus contributing to impaired $\mathrm{T}$ cell activation and proliferation with $\mathrm{T}$ cell tolerance $[54,55]$. These results indicate modulation of the host immune system to accommodate xenotransplanted cancer cell growth in the immunological tolerance model.

In conclusion, we developed a novel cancer xenograft model inzebrafish that has immunological tolerance and that will overcome the limitations of traditional xenotransplantation observed in immuno-compromised animals. Our dual RNA-seq 
analysis revealed the transcriptional networks underlying cancer-host interaction. This analysis has the potential to provide new therapeutic targets against cancer progression in the context of normal immunity. Thus, this model offers a useful platform for the evaluation of intact mechanisms of cancer progression and for zebrafish-based drug discovery. In addition, the immunological tolerance of this model will facilitate studies aimed at improving the success of organ transplantation.

\section{Acknowledgments}

All authors have read the journal's authorship agreement and conflict of interest policy. The authors have no potential conflict of interest to declare in relation to the contents of this manuscript.We thank S. Ichikawa for zebrafish breeding and maintenance, and R. Ikeyama and Y. Tamura for secretarial assistance. This work was supported in part by Grants-in-Aid for Scientific Research from the Japan Science and Technology Agency, KAKENHI, and the New Energy and Industrial Technology Development Organization, Japan.

\section{References}

[1] Ito M, Hiramatsu $\mathrm{H}$, Kobayashi $\mathrm{K}$, Suzue $\mathrm{K}$, Kawahata M, Hioki K, et al. NOD/SCID/gamma(c)(null) mouse: an excellent recipient mouse model for engraftment of human cells. Blood. 2002;100:3175-82.

[2] Steel GG, Courtenay VD, Rostom AY. Improved immune-suppression techniques for the exongrafting of human tumours. British journal of cancer. 1978;37:224-30.

[3] Shultz LD, Ishikawa F, Greiner DL. Humanized mice in translational biomedical research. Nature reviews Immunology. 2007;7:118-30.

[4] Fridman WH, Pages F, Sautes-Fridman C, Galon J. The immune contexture in human tumours: impact on clinical outcome. Nature reviews Cancer. 2012;12:298-306.

[5] Johnson JI, Decker S, Zaharevitz D, Rubinstein LV, Venditti JM, Schepartz S, et al. Relationships between drug activity in NCI preclinical in vitro and in vivo models and early clinical trials. British journal of cancer. 2001;84:1424-31.

[6] Dedrick RL, Morrison PF. Carcinogenic potency of alkylating agents in rodents and humans. Cancer research. 1992;52:2464-7.

[7] Lam SH, Wu YL, Vega VB, Miller LD, Spitsbergen J, Tong Y, et al. Conservation of gene expression signatures between zebrafish and human liver tumors and tumor progression. Nature biotechnology. 2006;24:73-5. 
[8] Sofia Vala I, Martins LR, Imaizumi N, Nunes RJ, Rino J, Kuonen F, et al. Low doses of ionizing radiation promote tumor growth and metastasis by enhancing angiogenesis. PLoS One. 2010;5:e11222.

[9] Nicoli S, Ribatti D, Cotelli F, Presta M. Mammalian tumor xenografts induce neovascularization in zebrafish embryos. Cancer research. 2007;67:2927-31.

[10] Kuroyanagi J, Shimada Y, Zhang B, Ariyoshi M, Umemoto N, Nishimura Y, et al. Zinc finger MYND-type containing 8 promotes tumour angiogenesis via induction of vascular endothelial growth factor-A expression. FEBS letters. 2014;588:3409-16.

[11] Rouhi P, Jensen LD, Cao Z, Hosaka K, Lanne T, Wahlberg E, et al. Hypoxia-induced metastasis model in embryonic zebrafish. Nature protocols. 2010;5:1911-8.

[12] Shimada Y, Nishimura Y, Tanaka T. Zebrafish-based systems pharmacology of cancer metastasis. Methods in molecular biology. 2014;1165:223-38.

[13] Yang XJ, Cui W, Gu A, Xu C, Yu SC, Li TT, et al. A novel zebrafish xenotransplantation model for study of glioma stem cell invasion. PLoS One. 2013;8:e61801.

[14] Lee HJ, Lowdon RF, Maricque B, Zhang B, Stevens M, Li D, et al. Developmental enhancers revealed by extensive DNA methylome maps of zebrafish early embryos. Nature communications. 2015;6:6315.

[15] Bertrand JY, Traver D. Hematopoietic cell development in the zebrafish embryo. Current opinion in hematology. 2009;16:243-8.

[16] Chen AT, Zon LI. Zebrafish blood stem cells. J Cell Biochem. 2009;108:35-42.

[17] Carradice D, Lieschke GJ. Zebrafish in hematology: sushi or science? Blood. 2008;111:3331-42.

[18] Meeker ND, Trede NS. Immunology and zebrafish: spawning new models of human disease. Developmental and comparative immunology. 2008;32:745-57.

[19] White RM, Sessa A, Burke C, Bowman T, LeBlanc J, Ceol C, et al. Transparent adult zebrafish as a tool for in vivo transplantation analysis. Cell stem cell. 2008;2:183-9.

[20] Stoletov K, Montel V, Lester RD, Gonias SL, Klemke R. High-resolution imaging of the dynamic tumor cell vascular interface in transparent zebrafish. Proc Natl Acad Sci U S A. 2007;104:17406-11.

[21] Tang Q, Abdelfattah NS, Blackburn JS, Moore JC, Martinez SA, Moore FE, et al. Optimized cell transplantation using adult rag2 mutant zebrafish. Nature methods. 2014;11:821-4.

[22] Zhang B, Shimada Y, Kuroyanagi J, Umemoto N, Nishimura Y, Tanaka T. Quantitative phenotyping-based in vivo chemical screening in a zebrafish model of leukemia stem cell xenotransplantation. PLoS One. 2014;9:e85439.

[23] Monte W. The Zebrafish Book: A guide for the laboratory use of zebrafish (Danio rerio). 4th edition. Acta neurochirurgica. 2000.

[24] Zhang B, Shimada Y, Kuroyanagi J, Nishimura Y, Umemoto N, Nomoto T, et al. Zebrafish xenotransplantation model for cancer stem-like cell study and high-throughput screening of inhibitors. Tumour biology : the journal of the International Society for Oncodevelopmental Biology and Medicine. 2014;35:11861-9.

[25] Zhang B, Shimada Y, Kuroyanagi J, Ariyoshi M, Nomoto T, Shintou T, et al. In vivo selective imaging and inhibition of leukemia stem-like cells using the fluorescent carbocyanine derivative, DiOC5(3). Biomaterials. 2015;52:14-25.

[26] Sun J, Nishiyama T, Shimizu K, Kadota K. TCC: an R package for comparing tag count data with robust normalization strategies. BMC bioinformatics. 2013;14:219.

[27] Schubert LA, Jeffery E, Zhang Y, Ramsdell F, Ziegler SF. Scurfin (FOXP3) acts as a repressor of transcription and regulates T cell activation. J Biol Chem. 2001;276:37672-9.

[28] Sakaguchi S, Sakaguchi N, Shimizu J, Yamazaki S, Sakihama T, Itoh M, et al. Immunologic tolerance maintained by $\mathrm{CD} 25+\mathrm{CD} 4+$ regulatory $\mathrm{T}$ cells: their common role in controlling autoimmunity, tumor immunity, and transplantation tolerance. Immunological reviews. 2001;182:1832.

[29] Chai JG, Xue SA, Coe D, Addey C, Bartok I, Scott D, et al. Regulatory T cells, derived from naive CD4+CD25- $\mathrm{T}$ cells by in vitro Foxp3 gene transfer, can induce transplantation tolerance. Transplantation. 2005;79:1310-6.

[30] Quintana FJ, Iglesias AH, Farez MF, Caccamo M, Burns EJ, Kassam N, et al. Adaptive autoimmunity and Foxp3-based immunoregulation in zebrafish. PLoS One. 2010;5:e9478.

[31] Subramanian A, Tamayo P, Mootha VK, Mukherjee S, Ebert BL, Gillette MA, et al. Gene set enrichment analysis: a knowledge-based approach for interpreting genome-wide expression profiles. Proc Natl Acad Sci U S A. 2005;102:15545-50.

[32] Kotelnikova E, Yuryev A, Mazo I, Daraselia N. Computational approaches for drug repositioning and combination therapy design. Journal of bioinformatics and computational biology. 2010;8:593-606. [33] Owen RD. Immunogenetic Consequences of Vascular Anastomoses between Bovine Twins. Science. 1945;102:400-1. 
[34] Southam CM. Immunologic tolerance to human cancer transplants in rats. Cancer research. 1966;26:2496-502.

[35] Zhang Y, Sharma JM. Immunological tolerance in chickens hatching from eggs injected with cellassociated herpesvirus of Turkey (HVT). Developmental and comparative immunology. 2003;27:431-8. [36] Liu WT, Li LH. Examination of immune tolerance to heterologous transplanted tumor of human stomach cancer in Syria Hamsters. Fish \& shellfish immunology. 2004;20:67-9.

[37] Meijer AH, Spaink HP. Host-pathogen interactions made transparent with the zebrafish model. Current drug targets. 2011;12:1000-17.

[38] Novoa B, Bowman TV, Zon L, Figueras A. LPS response and tolerance in the zebrafish (Danio rerio). Fish Shellfish Immunol. 2009;26:326-31.

[39] Lee LM, Seftor EA, Bonde G, Cornell RA, Hendrix MJ. The fate of human malignant melanoma cells transplanted into zebrafish embryos: assessment of migration and cell division in the absence of tumor formation. Developmental dynamics : an official publication of the American Association of Anatomists. 2005;233:1560-70.

[40] Corkery DP, Dellaire G, Berman JN. Leukaemia xenotransplantation in zebrafish--chemotherapy response assay in vivo. British journal of haematology. 2011;153:786-9.

[41] von Herrath MG, Harrison LC. Antigen-induced regulatory T cells in autoimmunity. Nature reviews Immunology. 2003;3:223-32.

[42] Khazaie K, von Boehmer H. The impact of CD4+CD25+ Treg on tumor specific CD8+ T cell cytotoxicity and cancer. Seminars in cancer biology. 2006;16:124-36.

[43] Onishi H, Morisaki T, Katano M. Immunotherapy approaches targeting regulatory T-cells. Anticancer research. 2012;32:997-1003.

[44] Zou W. Regulatory T cells, tumour immunity and immunotherapy. Nature reviews Immunology. 2006;6:295-307.

[45] Westermann AJ, Gorski SA, Vogel J. Dual RNA-seq of pathogen and host. Nature reviews Microbiology. 2012;10:618-30.

[46] Kolch W, Heidecker G, Lloyd P, Rapp UR. Raf-1 protein kinase is required for growth of induced NIH/3T3 cells. Nature. 1991;349:426-8.

[47] Falck Miniotis M, Arunan V, Eykyn TR, Marais R, Workman P, Leach MO, et al. MEK1/2 inhibition decreases lactate in BRAF-driven human cancer cells. Cancer research. 2013;73:4039-49.

[48] Zaidi MR, Merlino G. The two faces of interferon-gamma in cancer. Clinical cancer research : an official journal of the American Association for Cancer Research. 2011;17:6118-24.

[49] Janson PC, Marits P, Thorn M, Ohlsson R, Winqvist O. CpG methylation of the IFNG gene as a mechanism to induce immunosuppression [correction of immunosupression] in tumor-infiltrating lymphocytes. J Immunol. 2008;181:2878-86.

[50] Fan S, Meng D, Xu T, Chen Y, Wang J, Li X, et al. Overexpression of SLC7A7 predicts poor progression-free and overall survival in patients with glioblastoma. Med Oncol. 2013;30:384.

[51] Gu L, Vogiatzi P, Puhr M, Dagvadorj A, Lutz J, Ryder A, et al. Stat5 promotes metastatic behavior of human prostate cancer cells in vitro and in vivo. Endocrine-related cancer. 2010;17:481-93.

[52] Ahonen TJ, Xie J, LeBaron MJ, Zhu J, Nurmi M, Alanen K, et al. Inhibition of transcription factor Stat5 induces cell death of human prostate cancer cells. J Biol Chem. 2003;278:27287-92.

[53] Liao Z, Nevalainen MT. Targeting transcription factor Stat5a/b as a therapeutic strategy for prostate cancer. American journal of translational research. 2011;3:133-8.

[54] Torres-Aguilar H, Aguilar-Ruiz SR, Gonzalez-Perez G, Munguia R, Bajana S, Meraz-Rios MA, et al. Tolerogenic dendritic cells generated with different immunosuppressive cytokines induce antigenspecific anergy and regulatory properties in memory CD4+ T cells. J Immunol. 2010;184:1765-75.

[55] Nomura K, Miyagawa S, Ayukawa K, Soeda J, Taniguchi S, Kawasaki S. Inhibition of urokinasetype plasminogen activator delays expression of c-jun, activated transforming growth factor beta 1 , and matrix metalloproteinase 2 during post-hepatectomy liver regeneration in mice. Journal of hepatology. 2002;36:637-44. 


\section{Figure legends}

Figure 1. Immunological toleranceinduced by irradiated PC3-KOr cell implantation at the embryonic stage. (A) Schematic representation of two-round cancer cell xenotransplantation. (B) Representative images of cancer cell-implanted zebrafish larvae at $1 \mathrm{dpi}$ (day post implantation) and $7 \mathrm{dpi}$. The cancer cells (red) were detected in larvae injected with PC3-KOr cells, but not in those injected with Ir-PC3KOr cells at 7 dpi. (C) Representative images of cancer progression after the second implantationin adulthood with naive PC3-KOr cells(7dpi) in Ir-PC3-KOr zebrafish (embryonic implantation with Ir-PC3-KOr) and control fish (embryonic implantation with Matrigelvehicle).Left panels: the cell injection site in the trunk. Right panels: tail regions showing cancer cell migration.(D) Quantification of cancer cells in the tail region at 7 dpi after the second xenotransplantationin adult zebrafish. KOrfluorescence intensity was measured to determine cancer cell area. (E) Quantification of the cancer proliferation after the second xenotransplantation in adult zebrafish.Cancer cell proliferation was calculated as the ratio of KOr fluorescenceintensity in the tumour area relative to that at day 0 . Differences between the two groups were examined for statistical significance by independent Student's ttest. $\mathrm{n}=10 . * P<0.05,{ }^{* *} P<0.01$.

Figure 2. Immunological tolerance to the same but not different cancer cell lines.

(A) Representative images (the injection site is the same as that in Figure 1C)of xenotransplantation of the same (K562-KOr) or different (PC3-KOr)cell line into zebrafish(7 dpi and $14 \mathrm{dpi})$ that had beenxenotransplantedwith Ir-K562-KOr in their embryonic stage. Yellow framed areasindicate areas of magnification. (B) KusabiraOrange (KOr)mRNA expressionin the blood of zebrafish after the second cancer cell 
xenotransplantationin adulthood(28 dpi).For multiple comparisons, the assessments were analysed by ANOVA followed by the Bonferroni-Dunn multiple comparison test.n=3. $* P<0.05$.

Figure 3. Different gene expression profiles in the immunologically tolerantcancer xenografts.(A) Zebrafishfoxp 3 gene expression in the blood of 3-mpf zebrafish before and afterPC3-KOr cell implantation in adulthood (28dpi). n=3. ${ }^{*} P<$

$0.05, * * P<0.01$. (B) Gene expression fold differences of inflammatory factors $c d 3$, ifn- $\gamma$, and il-17 in the blood of 3-mpf zebrafish before and after PC3-KOr cell implantation (28dpi). $\mathrm{n}=3 .{ }^{*} P<0.05,{ }^{*} P<0.01$. (C) Expression of candidate genes was confirmed by q-PCR after PC3-KOr cell implantation (7dpi) in adult zebrafish. $\mathrm{n}=3 . * P<0.05, * * P<0.01$. Differences between the two groups were examined for statistical significance by independent Student's t-test.

Table 1. Prediction of immunological tolerance mechanism using SNEA 
Table 1. Prediction of immunological tolerance mechanism using SNEA

\begin{tabular}{|c|c|c|c|c|}
\hline & Gene set seed & Name & Measured neighbours & $P$ value \\
\hline \multirow[t]{3}{*}{$\underline{\text { Human }}$} & $M E K 1 / 2$ & $\begin{array}{l}\text { mitogen-activated protein kinase } \\
\text { kinase } 1 / 2\end{array}$ & BHLHE41, PLAGL1 & 0.02 \\
\hline & $I F N G$ & interferon gamma & $C 4 A, C 4 B, S L C 7 A 7, C H R M 2, E N P P 2$ & 0.03 \\
\hline & STAT5A & $\begin{array}{l}\text { signal transducer and activator of } \\
\text { transcription } 5 \mathrm{~A}\end{array}$ & $C E L, C 4 A$ & 0.03 \\
\hline \multirow[t]{5}{*}{ Zebrafish } & $K L F 1$ & Kruppel-like factor 1 & hbz, epor, hbe1, sptb, gatal, alas 2 & 7.14E-05 \\
\hline & MYOD1 & myogenic differentiation 1 & tnni2, actg2, ccna2, myl2, smyd1, ccnb1, actal, myl1, ccne1, myh4, tnnc2 & $3.17 E-04$ \\
\hline & $T B X 20$ & T-box 20 & actcl & $3.84 E-03$ \\
\hline & $E 2 F 1$ & E2F family of transcription factor 1 & $\begin{array}{l}\text { skp2, kif23, sival, cdc45, cdt1, ppm1d, plk1, bmi1, dnmt1, melk, ncapd2, } \\
\text { chek2, cdca8, ccna2, mcm10, ccnb1, fbxo5, plau, ccne1, stmn1, gmnn }\end{array}$ & $1.28 E-07$ \\
\hline & $E 2 F 3$ & E2F family of transcription factor 3 & gmnn, mcm10, cdt1, aurka, ccnb1, plau, ccnb2, ccne1, ccna2, cdc20, fbxo5 & $4.08 E-08$ \\
\hline
\end{tabular}


A

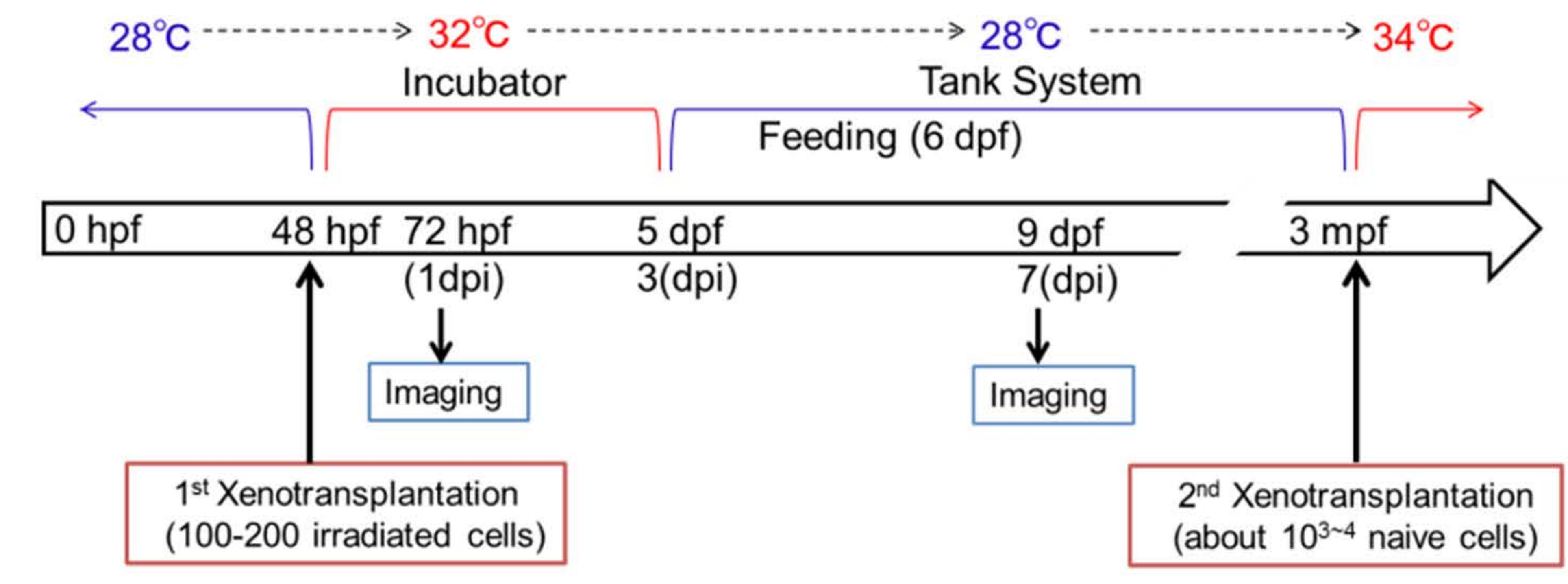

C

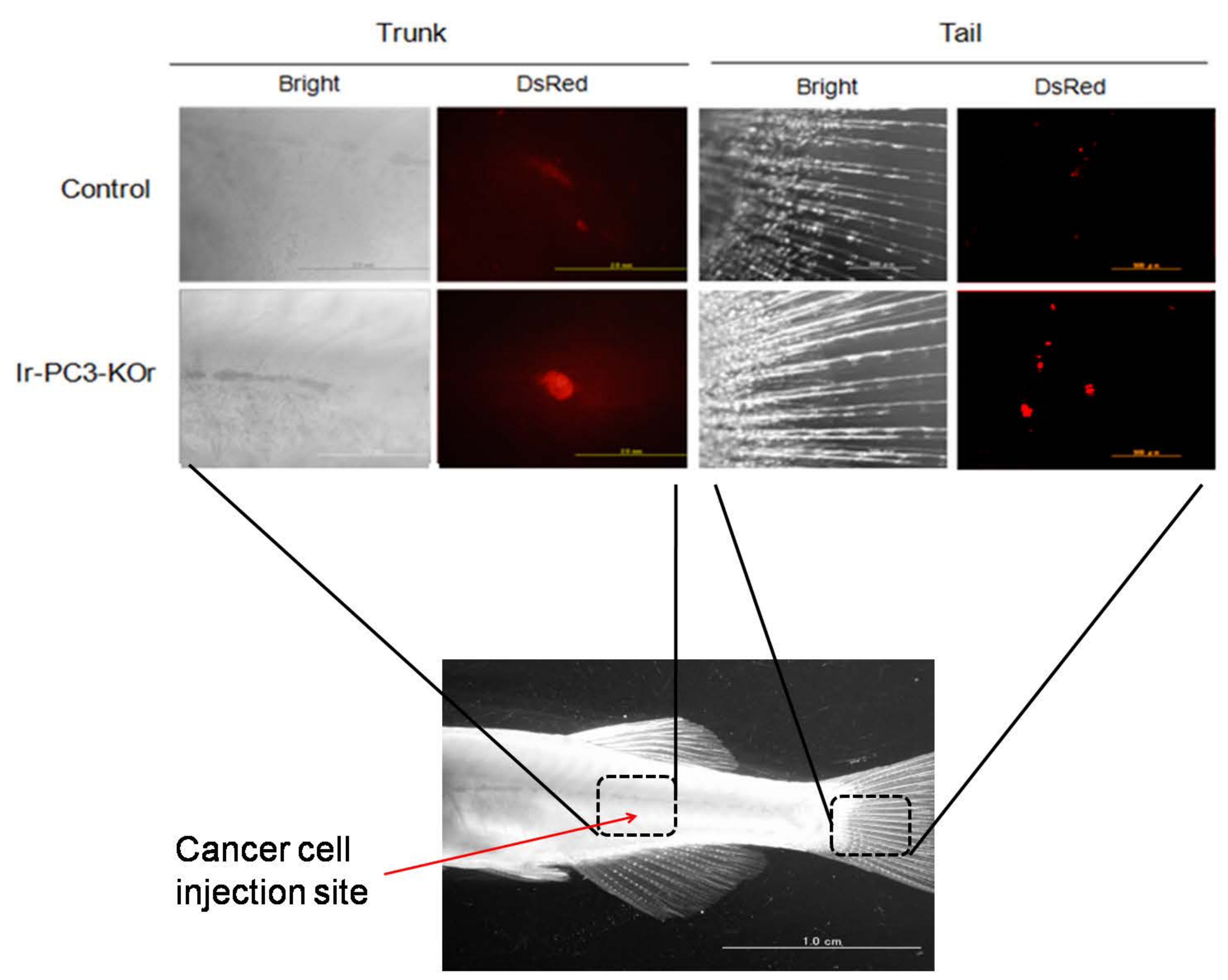

B

1 dpi
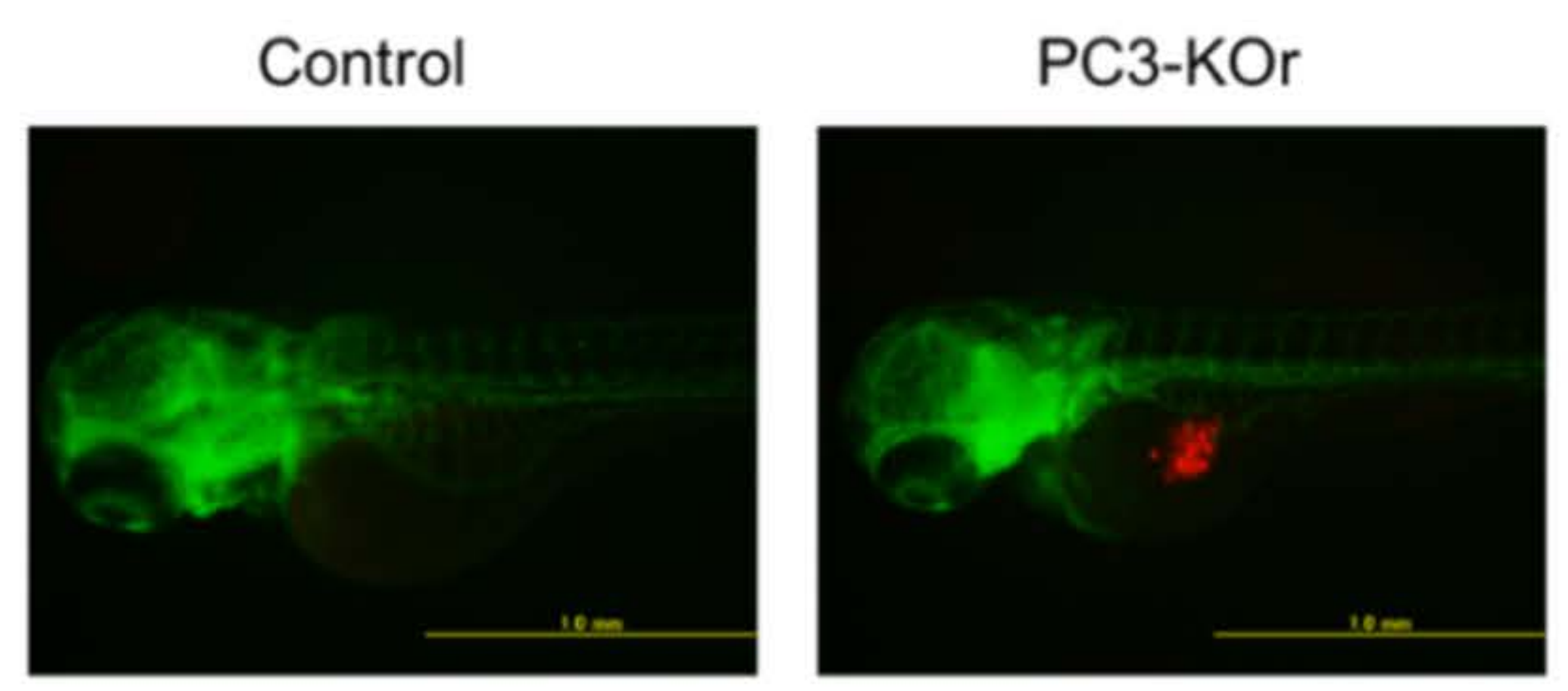

Ir-PC3-KOr

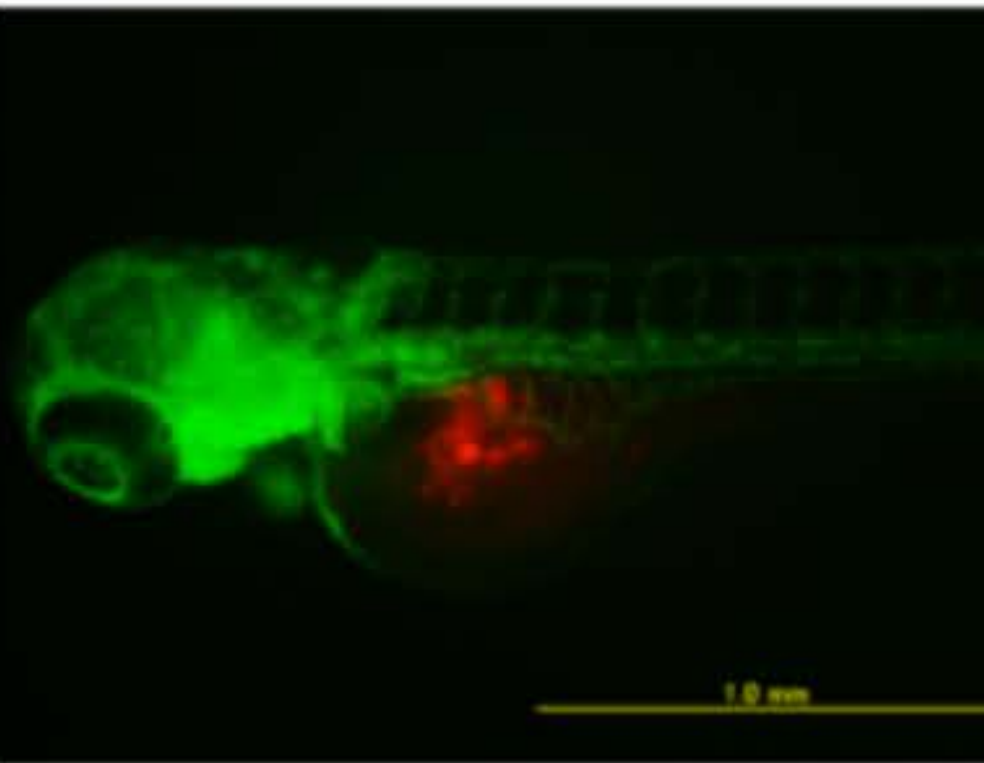

$7 \mathrm{dpi}$
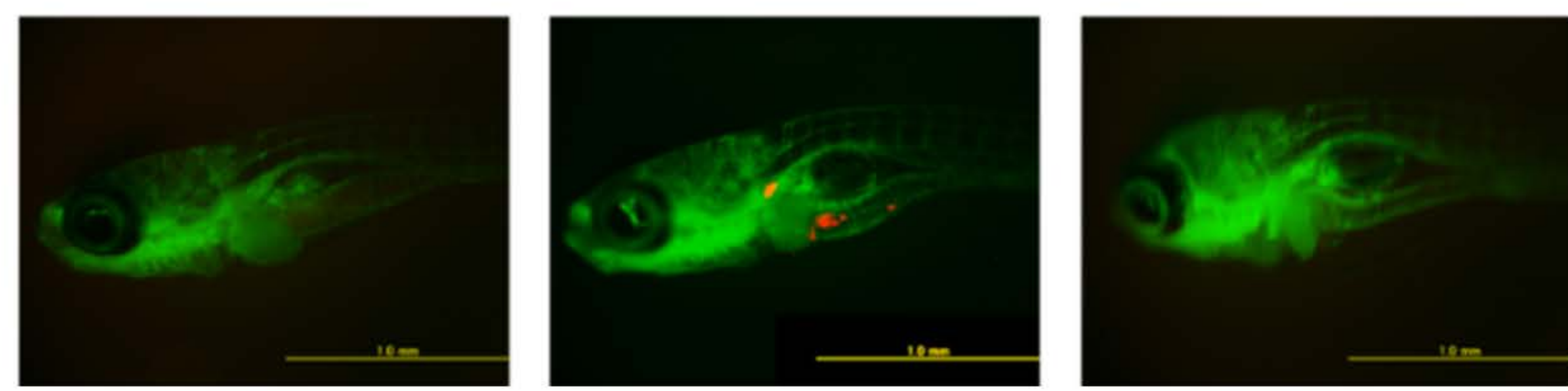

D

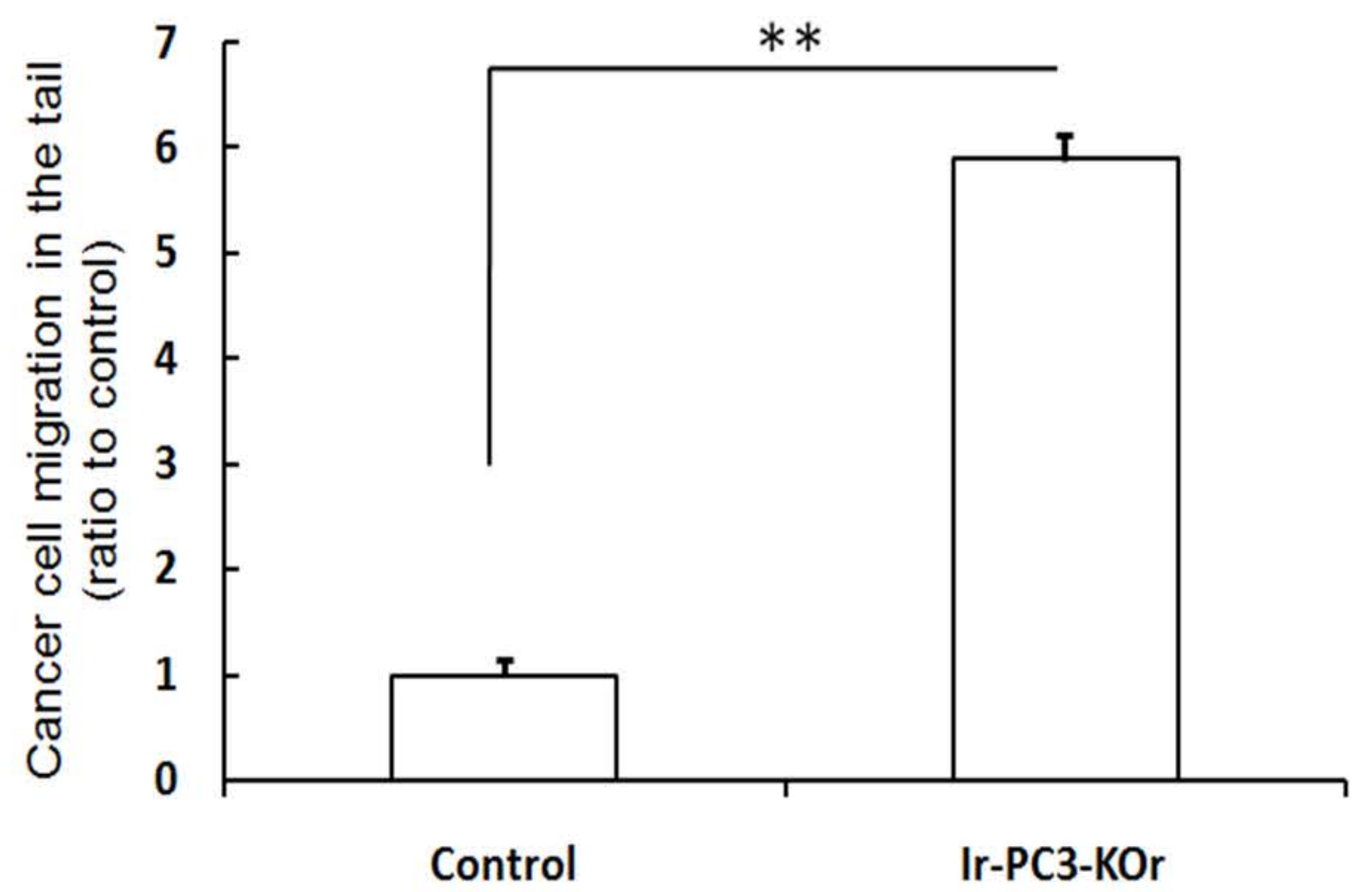

E

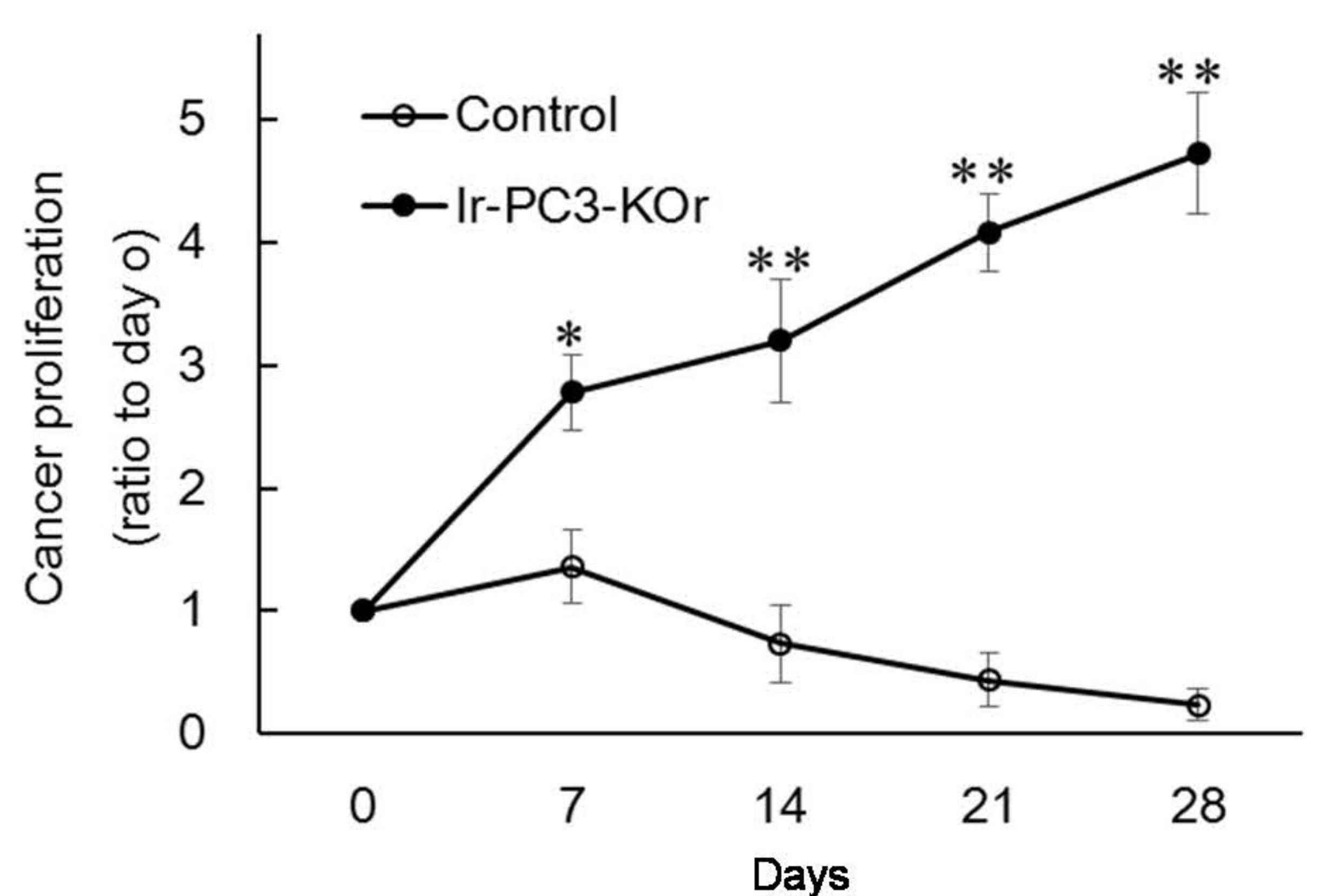


A

K562-KOr
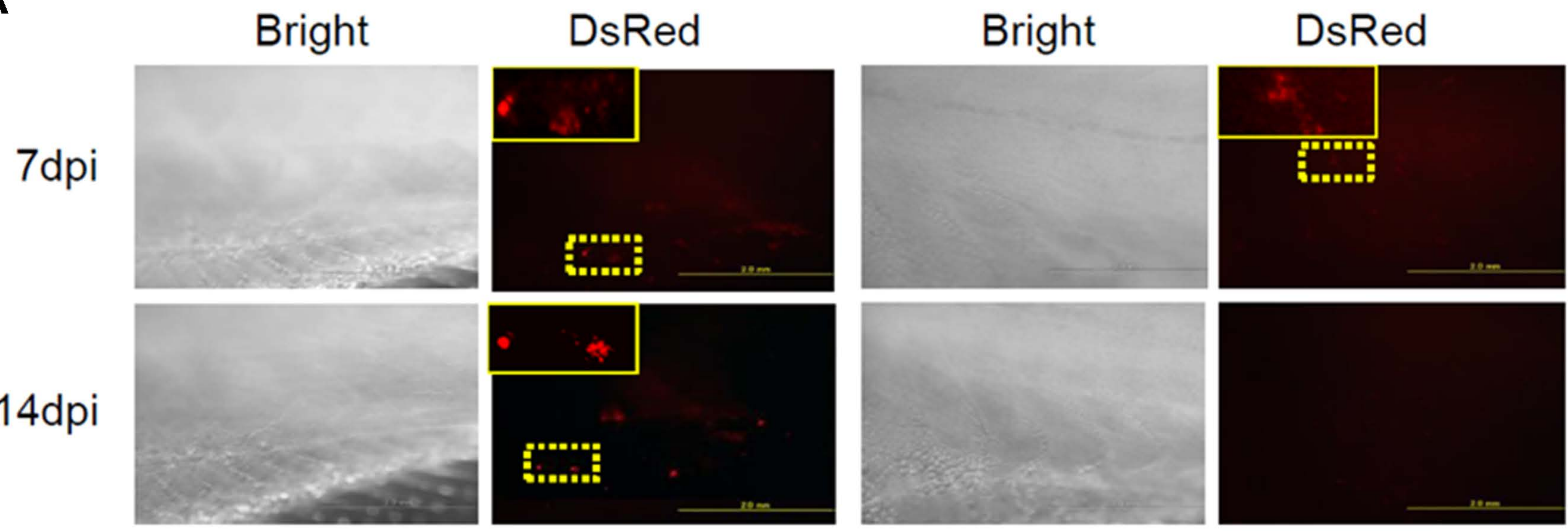

B

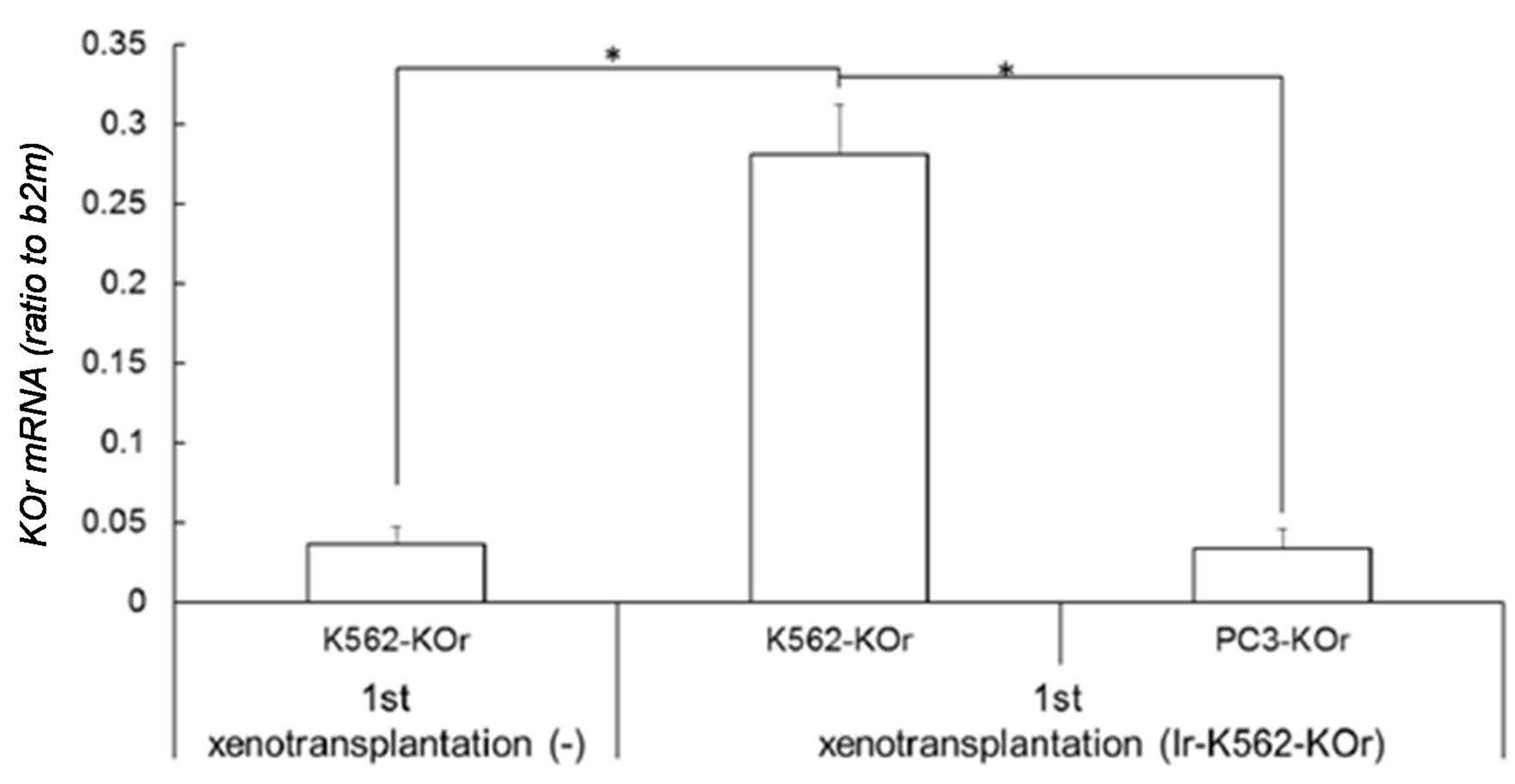


A

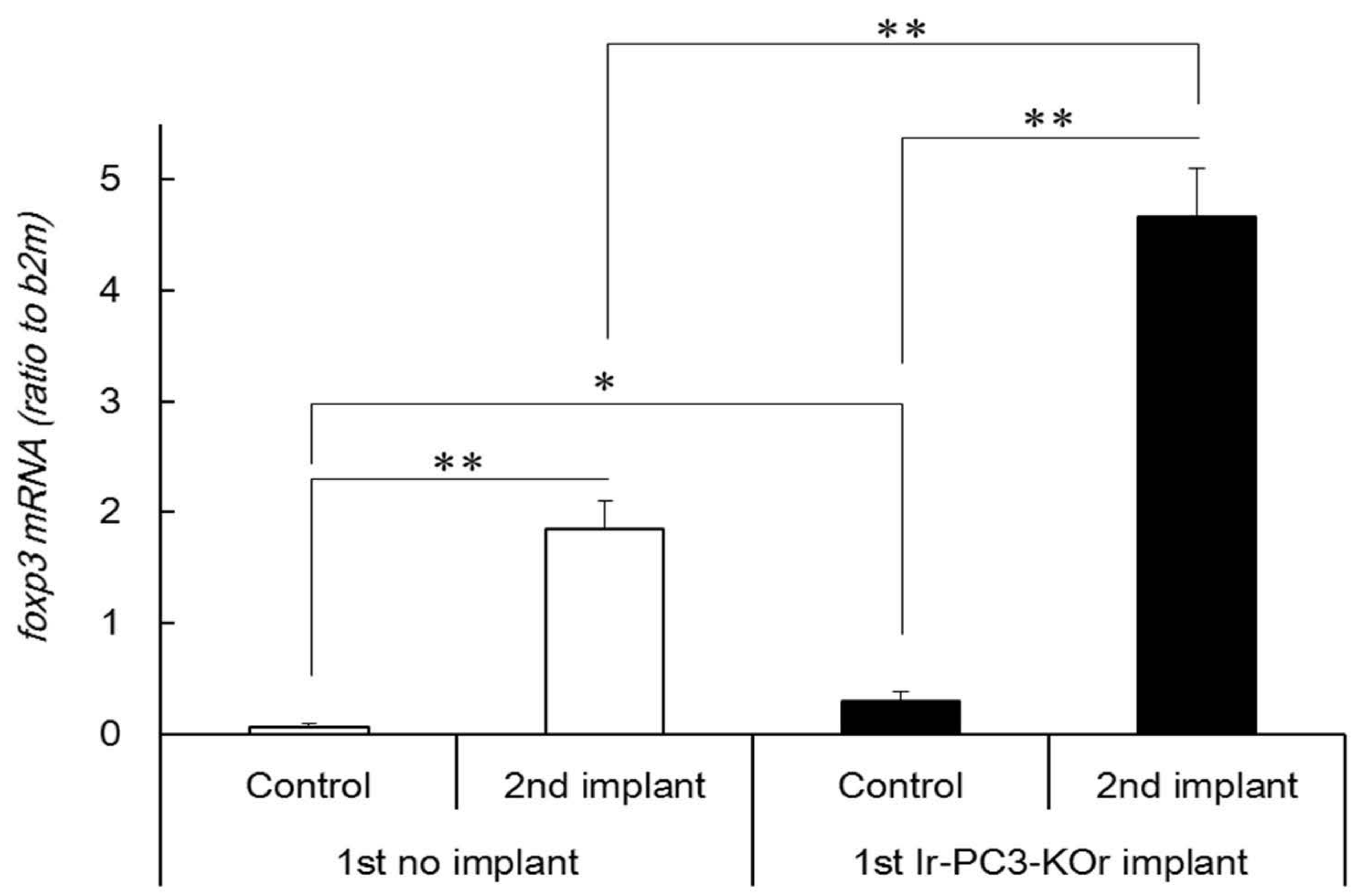

B
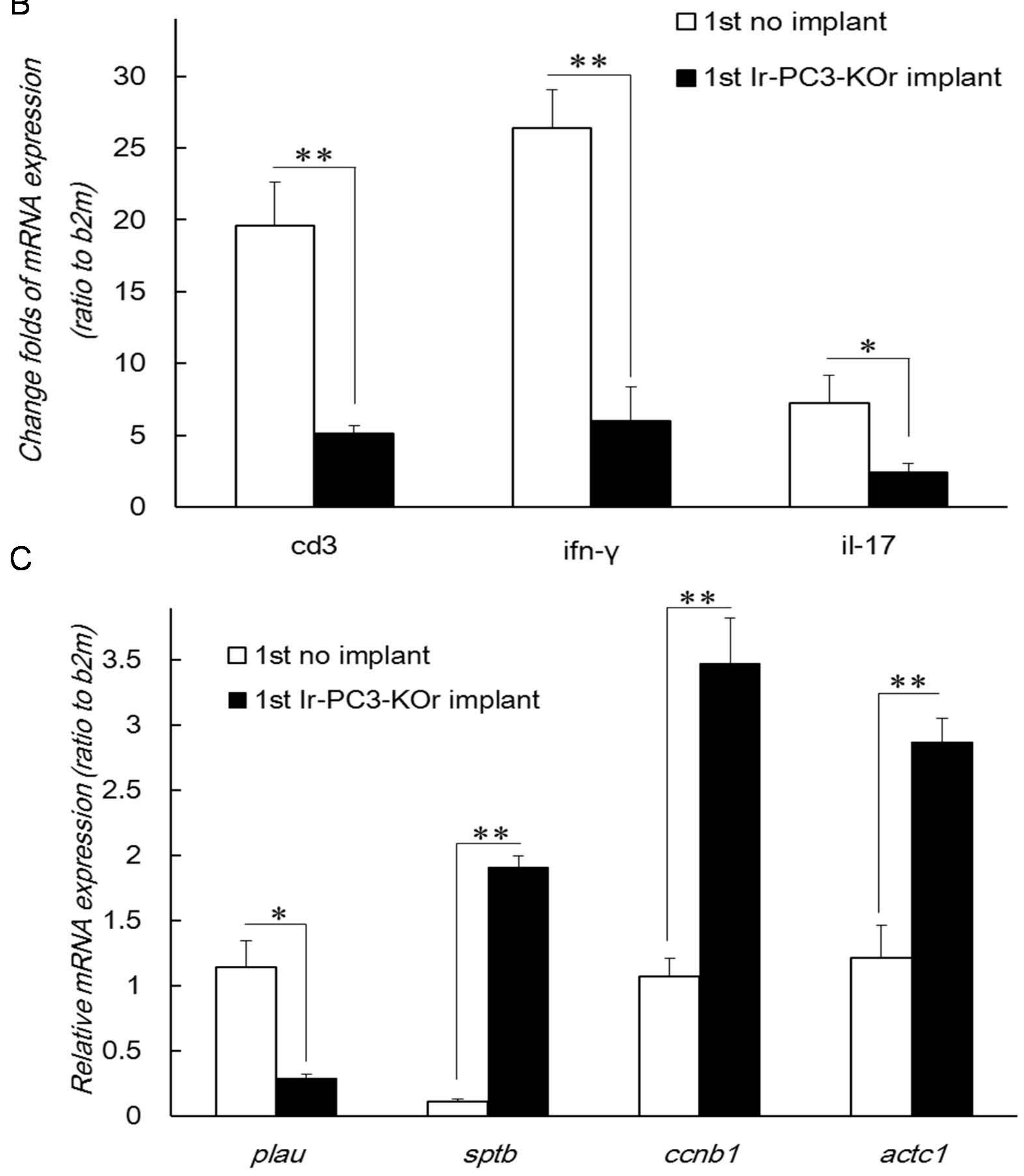